\title{
EchoGéo
}

18 | 2011

Varia

\section{Le "petit monde" des géographes}

Analyse des cartes mentales des pays du monde de 140 géographes présents au congrès UGI de Tunis en 2008

\section{Ali Bennasr et Claude Grasland}

\section{(2) OpenEdition}

\section{Journals}

\section{Édition électronique}

URL : https://journals.openedition.org/echogeo/12682

DOI : $10.4000 /$ echogeo.12682

ISSN : 1963-1197

\section{Éditeur}

Pôle de recherche pour l'organisation et la diffusion de l'information géographique (CNRS UMR 8586)

Référence électronique

Ali Bennasr et Claude Grasland, "Le "petit monde" des géographes », EchoGéo [En ligne], 18 | 2011, mis en ligne le 05 décembre 2011, consulté le 31 juillet 2021. URL : http://journals.openedition.org/ echogeo/12682 ; DOI : https://doi.org/10.4000/echogeo.12682

Ce document a été généré automatiquement le 31 juillet 2021.

EchoGéo est mis à disposition selon les termes de la licence Creative Commons Attribution - Pas d'Utilisation Commerciale - Pas de Modification 4.0 International (CC BY-NC-ND) 


\section{Le "petit monde" des géographes}

Analyse des cartes mentales des pays du monde de 140 géographes présents au congrès UGI de Tunis en 2008

\section{Ali Bennasr et Claude Grasland}

\section{NOTE DE L'ÉDITEUR}

Le questionnaire utilisé est disponible en annexe 1.

Certaines des illustrations sont peu lisibles à l'écran aussi vous sont-elles proposées pour impression en format PDF en annexe 2.

Nous remercions le Président de l'Association des géographes tunisiens et le comité d'organisation du congrès UGI 2008 de Tunis qui nous ont permis de réaliser l'enquête auprès des membres de l'UGI. Notre reconnaissance va également à l'ensemble des membres du laboratoire SYFACTE de l'Université de Sfax qui ont contribué à la réalisation pratique de l'enquête. Nous remercions les deux rapporteurs anonymes de la revue Echogeo pour leurs conseils et suggestions d'amélioration de la première version. Enfin, nous rappelons que les extraits de l'enquête EuroBroadMap qui sont cités en contrepoint de l'enquête UGI de Tunis font partie du projet FP7 EuroBrodMap - Visions of Europe SeenfromOutside, qui est financé par la DG Research de la Commission Européenne. Site web:http://www.eurobroadmap.eu

\section{Introduction}

\section{Les représentations du monde (en général) et de l'Europe (en particulier)}

1 Les représentations mentales du monde jouent un rôle crucial dans la construction des discours politiques des États mais aussi dans l'élaboration du travail scientifique en géographie (Lynch, 1960 ; Gould \& White, 1974 ; Moles \& Rohmer, 1978 ; Belhedi 1981 ; Paulet, 2002 ; Cauvin, 1999, 2002). L'union européenne apparaît de ce point de vue comme particulièrement originale puisque chacun des États membres porte une vision 
spécifique du monde héritée de traditions historiques et diplomatiques. Et il n'est dès lors pas évident qu'une vision collective soit encore fermement constituée sur ce qu'est ou ce que pourrait être la place de l'union européenne dans le monde (Walker, 2000).

Une étude réalisée pour la direction de la politique régionale (DG Regio) de la Commission Européenne en 2005-2007 dans le cadre du programme ESPON avait ainsi montré que les chercheurs et décideurs politiques européens ont encore des visions très classiques du découpage du monde (par continents) et des limites de l'Europe (importance de la coupure méditerranéenne, incertitudes sur la Turquie et la Russie). Or, les évolutions récentes du monde appellent des réponses plus ambitieuses que les replis frileux sur un continent européen aux limites finalement arbitraires. Publiés sous la forme d'une synthèse politique en anglais (Beckouche \& Grasland, 2008) puis d'un atlas scientifique en français (Didelon C., Grasland C., Richard Y., 2009), les résultats de cette première étude appelaient une analyse plus poussée des visions du monde croisant à la fois les points de vue des habitants de l'union européenne et ceux des autres pays du monde, dans une perspective non eurocentrique.

\section{Une enquête test sur les représentations de l'Europe dans le monde}

L'annonce en 2008 de l'obtention d'un financement de la DG recherche dans le cadre du $7^{e}$ PCRD (projet EuroBroadMap ${ }^{1}$, 2009-2011) a ouvert la possibilité d'approfondir ces premiers résultats en permettant de financer une enquête de grande portée sur les visions de l'Europe dans le monde. Mais avant de lancer une telle étude, il convenait de s'assurer que le questionnaire préalablement testé uniquement sur des chercheurs et décideurs politiques européens pourrait être rempli par des étudiants de pays aussi différents que la France, la Turquie, le Brésil, la Chine, l'Inde, le Cameroun, la Russie, ... En effet, à la différence des grandes enquêtes fondées sur des "sketch maps ", c'est à dire sur le tracé des pays connus sur un document papier vierge (Saarinen 1987, 1988a, 1988b ; Saarinen \& Mac Cabe, 1995), nous voulions tester une autre approche visant à comprendre le tracé des découpages en continents ou régions du monde. Nous voulions également tenter de mesurer l'attractivité des pays du monde pour les étudiants (ACA, 2005 ; Fabry, 2007) en utilisant le filtre des États et en exploitant les réponses à des questions plus classiques sur les lieux attractifs et répulsifs ainsi que sur la perception des distances.

4 Le congrès UGI qui s'est tenu à Tunis en 2008 est donc arrivé à point nommé, à un moment où le financement de l'enquête mondiale était déjà acquis mais où nous disposions de quelques mois de délais avant son lancement. Trouver réunis en un même lieu des géographes de la plupart des pays du monde nous semblait une occasion unique d'affiner nos questions. Le congrès UGI a donc servi de test pour l'élaboration d'un questionnaire sur les représentations de l'Europe dans le monde qui a été administré ensuite à près de 10000 étudiants de toutes disciplines localisés dans 42 villes de 18 pays du monde dans le cadre du projet EuroBroadMap financé par le $7^{\mathrm{e} P C R D T}$. Nous savions que ce futur questionnaire EuroBroadMap comporterait plusieurs questions potentiellement difficiles pour des non-géographes (e .g. découper le monde en régions, tracer des limites de l'Europe, citer des pays ou des villes, évaluer des distances ...) et nous voulions en évaluer la faisabilité sur une population a priori très favorable: les géographes présents au congrès de l'Union Géographique Internationale. L'hypothèse sous-jacente de cette phase test était que les questions 
auxquelles les géographes professionnels ne sauraient pas répondre étaient évidemment à bannir pour des étudiants non géographes (nous avons ainsi retiré une question sur les distances entre plusieurs villes du monde au vu des résultats très décevants de l'enquête UGI). L'intérêt du colloque UGI était aussi de disposer d'un échantillon comportant un grand nombre de nationalités, afin d'examiner les effets possibles du choix de la langue sur les réponses aux questions (nous avons ainsi conclu que l'usage unique de l'anglais ou du français n'était pas souhaitable et qu'il valait mieux rédiger les questionnaires dans la langue de chaque pays d'enquête sur les étudiants).

\section{Un test de l'originalité (ou la banalité) des visions du monde des géographes professionnels}

5 Réaliser une enquête sur les visions du monde des participants au congrès UGI de 2008 offrait toutefois un autre intérêt, peut-être d'ordre plus épistémologique, qui était d'examiner dans quelle mesure les géographes de métier proposent des visions du monde originales et échappant aux stéréotypes véhiculés par la presse et les médias. Avouons-le, nous espérions que les réponses des membres de notre corporation disciplinaire feraient preuve de plus de subtilité dans leur vision du monde que ces journalistes et hommes politiques européens qui expliquent doctement que «la Turquie n'est pas en Europe, c'est la géographie qui le dit ». Nous escomptions que dans leur découpage $\mathrm{du}$ monde, ils utiliseraient des catégories variées (aires culturelles, géopolitiques, économiques, climatiques ...) plutôt que de fausses évidences telles que les continents qui sont avant tout le fruit de l'histoire et de l'idéologie (Grataloup C., 2009). Enfin et surtout, nous pensions que la diversité des participants au congrès de l'UGI et la variété de leurs domaines d'intérêt aboutiraient à l'élaboration collective d'une vision du monde subtile et multidimensionnelle...

6 Disons d'entrée de jeu que notre espoir a été dans l'ensemble déçu et que la plupart des découpages du monde proposés par les géographes professionnels de l'UGI en 2008 ne sont guère différents de ce que nous avons pu observer ensuite en dépouillant l'enquête mondiale de 2009-2010 sur les étudiants non géographes. Mais les résultats les plus étonnants ne concernent pas tant la question des découpages du monde qu'une autre question, en apparence plus banale, qui consiste à demander aux personnes enquêtées de citer 5 pays du monde où elles aimeraient vivre dans un futur proche et cinq autres où elles ne le souhaiteraient pas. C'est sur l'exploitation des réponses à cette dernière question que portera le présent article, en s'interrogeant sur l'originalité ou au contraire la banalité de la vision du monde exprimée par les géographes présents au congrès UGI de 2008.

7 Cette question revêt un intérêt particulier dans la mesure où elle met en jeu deux processus mentaux très différents. D'un côté la connaissance des pays (positivement ou négativement on ne cite que ce que l'on connaît) et de l'autre côté leur niveau perçu d'attraction-répulsion tel qu'il peut se déduire des parts relatives des personnes qui souhaiteraient y vivre ou ne pas y vivre. Si les géographes professionnels ont par définition une connaissance a priori plus large des pays du monde que le reste de la population, rien ne prouve qu'ils mettent en jeu ces connaissances professionnelles dans le cadre d'une question qui est formulée de façon volontairement floue. 


\section{Un échantillon de 140 géographes présents au congrès UGI de 2008}

8 L'Union Géographique Internationale est une organisation très particulière dont le fonctionnement en congrès, organisés depuis 1922, a fait l'objet de recherches approfondies. Comme le rappelle P. Pinchemel dans l'avant-propos de l'ouvrage de synthèse qui lui a été consacré en 1996 : « son histoire s'inscrit dans des contextes difficiles: deux guerres mondiales et un court entre-deux guerres, une guerre froide, les guerres de libération et de décolonisation, les bouleversements démographiques, économiques et sociaux..., les révolutions idéologiques, culturelles, techniques ... » (Robic, Briend \& Rössler, 1996). Au cours de son histoire, l'UGI a toujours été un miroir fidèle des tensions politiques mondiales: "La contradiction apparaît permanente entre les volontés affirmées d'internationalisme, d'universalisme, et les revendications nationalistes, continentalistes, idéologiques, les oppositions entre les riches et les pauvres, le Nord et le Sud, entre les États européens et le reste du monde, entre les géographes du monde blanc, anglo-saxon, protestant et les autres pays du monde ». (ibid.). Cette situation n'est pas sans rappeler celle des Jeux Olympiques.

9 L'UGI contemporaine n'est évidemment pas un miroir de l'ensemble de la géographie mondiale et il était bien visible au colloque de l'UGI 2008 que la moyenne d'âge était sensiblement plus élevée que dans d'autres colloques et que les hommes étaient en proportion plus importante que les femmes. Grâce au soutien des organisateurs du congrès (UGI, Association des Géographes Tunisiens), nous avons été autorisés à collecter des questionnaires pendant les quatre journées du congrès de l'UGI. Pour s'assurer d'un échantillon représentatif, il aurait été souhaitable de distribuer systématiquement le questionnaire à tous les participants et de vérifier le taux de réponse par catégorie, mais cela était impossible en raison du faible nombre de séances plénières et d'horaires très contraignants. Nous avons pu cependant administrer le questionnaire en contact face à face dans les stands d'exposition, ce qui permettait de mieux contrôler le temps de réponse (en principe limité à 15 minutes) et évaluer les difficultés rencontrées par les participants. Le stand du laboratoire de recherche tunisienne SYFACTE, les autres stands d'exposition des délégations nationales, la cafétéria ... sont les principaux lieux où a pu être réalisée l'enquête, dans une ambiance très conviviale et sympathique. Les questionnaires étaient proposés au choix en français ou en anglais par des enquêteurs eux-mêmes français ou tunisiens mais parlant anglais. Nous avons essayé de cibler le maximum de nationalités différentes mais il y a eu à l'évidence des effets de groupe ou de réseaux de connaissance favorisant la présence des nationalités des enquêteurs dans les réponses.

Comme on peut le voir dans le tableau 1, les 140 questionnaires recueillis révèlent une population relativement âgée (la moitié des personnes enquêtées avaient plus de 47 ans) et au sex-ratio déséquilibré (à peu près deux tiers d'hommes et un tiers de femmes). Ceci reflète sans doute assez fidèlement la structure des membres de l'UGI et montre que, même si les femmes ne sont plus cantonnées au rôle d'accompagnatrices et au « ladies program » de jadis, des progrès restent encore à accomplir (Robic, 1997) ... 
Tableau 1 - Enquêtés par date de naissance et genre

\begin{tabular}{|l|l|l|l|l|l|l|}
\hline & $1978-1982$ & $1968-1977$ & $1958-1967$ & $1948-1957$ & avant 1948 & Total \\
\hline & $(\mathbf{3 0 - 3 5}$ ans $)$ & $(\mathbf{3 5 - 4 0}$ ans $)$ & $(\mathbf{4 0 - 5 0}$ ans $)$ & $(\mathbf{5 0 - 6 0}$ ans $)$ & $(+\mathbf{6 0}$ ans $)$ & \\
\hline Hommes & 18 & 13 & 15 & 31 & 12 & 89 \\
\hline Femmes & 10 & 9 & 15 & 11 & 6 & 51 \\
\hline Total & 28 & 22 & 30 & 42 & 18 & 140 \\
\hline
\end{tabular}

11 En ce qui concerne l'origine géographique des personnes enquêtées, nous avons retenu dans le tableau 2 comme critère d'analyse le lieu de résidence actuel plutôt que la nationalité ou le lieu de naissance. On voit que sur les 140 réponses, à peu près le quart correspond à des résidents tunisiens (37 sur 140).

Tableau 2 : Enquêtés par lieu de résidence en 2008

\begin{tabular}{|c|c|c|c|c|c|c|c|c|}
\hline TUN & EUE & & EUO & & DEV & & RIC & \\
\hline Tunisie & \multicolumn{2}{|c|}{ Europe de l'Est } & \multicolumn{2}{|c|}{ Europe de l'Ouest } & \multicolumn{2}{|c|}{ Autres PVD } & \multicolumn{2}{|c|}{ Autres riches } \\
\hline & Pologne & 5 & Allemagne & 12 & Russie & 5 & USA & 10 \\
\hline & Roumanie & 5 & France & 11 & Chine & 4 & Israel & 5 \\
\hline & Rep. Tch. & 2 & Espagne & 5 & Algérie & 2 & Corée Sud & 5 \\
\hline & Croatie & 2 & Roy. Uni & 5 & Brésil & 2 & Japon & 1 \\
\hline & Lettonie & 2 & Italie & 4 & Egypte & 2 & Canada & 1 \\
\hline & Hongrie & 1 & Pays-Bas & 2 & Iran & 1 & & \\
\hline & Lithuanie & 1 & Belgique & 1 & Nigéria & 1 & & \\
\hline & Slovénie & 1 & Finlande & 1 & Af. du Sud & 1 & & \\
\hline & & & Irlande & 1 & & & & \\
\hline & & & Norvège & 1 & & & & \\
\hline & & & Portugal & 1 & & & & \\
\hline 37 & & 19 & & 44 & & 18 & & 22 \\
\hline
\end{tabular}

12 La comparaison entre la distribution des questionnaires recueillis et le registre des inscriptions au congrès UGI 2008 montre que l'enquête a permis de toucher environ $1 / 8^{e}$ (13\%) des présents. Le nombre élevé de questionnaires émanant de géographes 
tunisiens ne fait que refléter la présence massive de ces derniers lors du congrès organisé par leur pays. En revanche, il y a clairement un biais en faveur des géographes européens qui sont surreprésentés ( $49 \%$ des questionnaires contre $31 \%$ des participants) tandis que les géographes asiatiques sont sous-représentés ( $11 \%$ des questionnaires contre $26 \%$ des participants (tableau 2)

Tableau 3 - Taux de couverture de la population présente

\begin{tabular}{|l|l|l|l|l|l|l|}
\hline & \multicolumn{2}{|l|}{ Participants (A) } & \multicolumn{2}{l|}{ Enquête (B) } & \multicolumn{2}{l|}{ Représentativité (A/B) } \\
\hline & effectif & $\%$ & effectif & $\%$ & proportion & équilibre \\
\hline Tunisie & 285 & $26 \%$ & 37 & $26 \%$ & 0,13 & 1,0 \\
\hline reste de l'Afrique & 63 & $6 \%$ & 6 & $4 \%$ & 0,10 & 0,8 \\
\hline Europe & 342 & $31 \%$ & 68 & $49 \%$ & 0,20 & 1,6 \\
\hline Asie & 284 & $26 \%$ & 16 & $11 \%$ & 0,06 & 0,4 \\
\hline Amérique du Nord & 81 & $7 \%$ & 11 & $8 \%$ & 0,14 & 1,1 \\
\hline Amérique du Sud & 26 & $2 \%$ & 2 & $1 \%$ & 0,08 & 0,6 \\
\hline \hline Océanie & 32 & $3 \%$ & 0 & $0 \%$ & 0,00 & 0,0 \\
\hline \hline Total & $\mathbf{1 1 1 3}$ & $\mathbf{1 0 0} \%$ & $\mathbf{1 4 0}$ & $\mathbf{1 0 0} \%$ & $\mathbf{0 , 1 3}$ & $\mathbf{1 , 0}$ \\
\hline
\end{tabular}

13 Notre enquête correspond donc dans ses grandes lignes à l'échantillon des géographes présents au congrès UGI de Tunis, mais celui-ci ne reflète pas pour autant la communauté des géographes et la présence des pays varie fortement d'un congrès UGI à l'autre. Le congrès de l'UGI 2008 fut à cet égard marqué par une présence beaucoup plus massive des pays arabes et musulmans, en raison de son lieu d'implantation et du fait que plusieurs exposés en séance plénière furent prononcés en langue arabe avec traduction. Deux ans après, la conférence UGI qui se tenait à Tel Aviv fut largement boycottée par ces mêmes délégations arabes mais aussi par une partie des autres pays du monde. L'inégalité économique pèse également lourd dans la présence des délégations car comme le soulignait cruellement P. Pinchemel en 1996, «l'UGI se veut internationale mais la réalité apparaît différente. Beaucoup d'États ne sont pas encore présents et parmi les États membres trop d'entre eux ne le sont que virtuellement, sans parler de ceux qui en sont exclus pour cause de défaillance financière » (Robic \& al., 1996).

Toutes ces réserves étant posées, quelle exploitation peut-on faire des résultats obtenus lors de l'enquête UGI de Tunis en 2008 ? Il nous semble que la seule manière valable d'appréhender correctement les résultats sera de comparer les résultats à l'aide des trois ou cinq groupes de réponses dont la composition est indiquée dans le tableau 2 : Les géographes tunisiens (TUN $=37)$; les géographes des pays membres de l'union européenne $(\mathrm{EU}=62)$ que l'on peut décomposer en ancien pays membres d'Europe de l'Ouest $(\mathrm{EUO}=44)$ et nouveaux pays membres ou candidats d'Europe Centre orientale $(E U E=19)$; et enfin les géographes des autres pays du monde qui forment un groupe 
assez hétérogène mais que l'on peut affiner en séparant les pays riches (RIC $=22)$ et les pays émergents ou en développement $(\mathrm{DEV}=18)$. Même si ces catégories sont assez grossières, elles permettent d'explorer ce qui dans les réponses relève de comportements universels (même réponse dans chacun des groupes) et ce qui est au contraire spécifique (réponses différentes d'un groupe à l'autre).

\section{Évaluation globale de la connaissance et de l'attractivité des pays du monde}

15 A la question classique «En dehors de votre propre pays, citez 5 pays dans lesquels vous aimeriez vivre / 5 pays dans lesquels vous n'aimeriez pas vivre ", les géographes qui ont répondu au questionnaire ont apporté des réponses très diversifiées. Pas moins de 110 États ont été cités sur les 150 à 200 que compte le monde. Mais cette diversité ne semble pas être beaucoup plus élevée que celle des réponses collectées ultérieurement sur des échantillons d'étudiants non-géographes. Si l'on excepte quelques réponses concernant des territoires supranationaux ("Pays africains", "Pays du Golfe", «Maghreb », ...) ou infra-nationaux ("Ecosse», «sud-ouest des États-Unis», ...), la plupart des personnes ont accepté la contrainte imposée de répondre à travers la grille des États. Ce n'est que de façon exceptionnelle que les enquêtés ont refusé de répondre. Plus précisément, presque tous ont accepté de citer les pays où ils aimeraient vivre (sauf une personne qui a répondu "Anywhere») mais un nombre sensiblement plus élevé a refusé de répondre à la question suivante des pays où ils n'aimeraient pas vivre, la considérant sans doute comme politiquement incorrecte ou impertinente.

16 L'un des grands intérêts méthodologique de l'enquête pilote menée à l'UGI a été de montrer que le fait pour un pays d'être cité plutôt qu'ignoré était tout aussi important dans une première phase de l'analyse que l'étude de la part relative des avis positifs et négatifs. Pour prendre un exemple typique de résultat, la Corée du Sud est plus appréciée que la Corée du Nord, mais elle n'a été citée que 4 fois ( 3 positivement et une négativement) alors que la seconde l'a été 14 fois (toutes négativement). On peut donc dire que la Corée du Nord est un pays beaucoup plus structurant des représentations mentales des personnes enquêtées que la Corée du Sud.

Comme on peut le voir sur la figure 1 en examinant la taille des cercles, les pays les plus cités (positivement ou négativement) dans les 140 questionnaires sont la France et les États-Unis (73 chacun), seuls pays qui apparaissent dans plus de la moitié des réponses. Viennent ensuite le Canada (66), l'Italie (53), l'Espagne (40), la Grande Bretagne (39), la Chine et la Russie ( 36 chacune) qui sont citées par au moins une personne sur quatre. L'Allemagne n'arrive qu'en 9e position (33) suivie par l'Australie et l'Irak (30) puis l'Arabie Saoudite et l'Iran (29), la Suède (24), l'Afrique du Sud (23), l'Afghanistan et Israël (22), la Nouvelle-Zélande et l'Inde (18), le Japon et les Pays-Bas (17), la Norvège (16), la Tunisie (15), la Corée du Nord et la Libye (14). 
Figure 1 - Distribution des pays cités par 140 membres du congrès UGI Tunis 2008

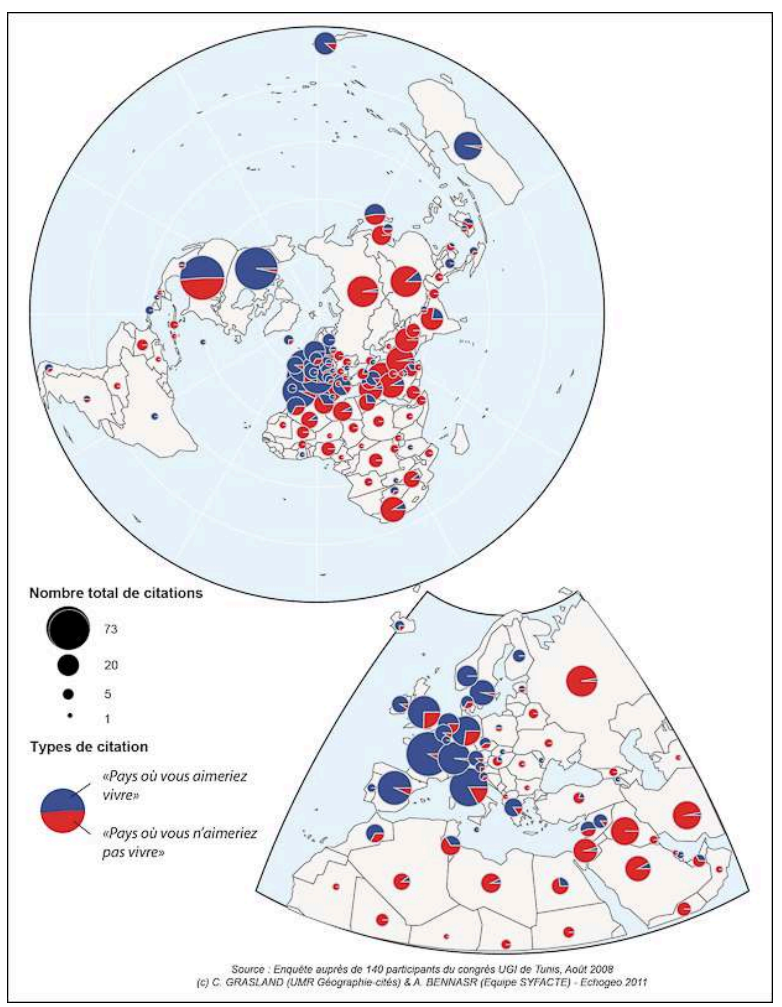

Source : Bennasr A. (Syfacte, Université de Sfax) \& Grasland C.(CNRS, Université Paris Diderot), enquête auprès de 140 participants du congrès UGI de Tunis, août 2008.

Si la notoriété permet de définir les pays les plus structurants dans la représentation du monde, elle doit donc être complétée par la prise en compte de l'attraction ou de la répulsion qu'exercent les pays les plus cités. On pourra ainsi différencier les pays qui sont presque toujours cités positivement (Canada, Australie, ...), ceux qui sont presque toujours cités négativement (Iran, Chine, Russie) et enfin ceux, plus rares, qui récoltent à la fois des appréciations positives et négatives (États-Unis, Japon, Allemagne). Un indice d'attractivité dérivé de l'analyse des migrations permet d'évaluer ce degré d'attraction ou de répulsion sous la forme d'un indice simple variant entre -1 (toutes les appréciations sont négatives et +1 (toutes les appréciations sont positives). On complète l'indice d'attractivité par un indice de notoriété qui divise le nombre de citations d'un pays par le nombre de personnes ayant pu le citer. Cet indice de connaissance tient compte du fait que les personnes ne pouvaient pas citer leur propre pays (encadré 1). 
Encadré 1 : Calcul des indices d'attractivité et de notoriété des pays du monde

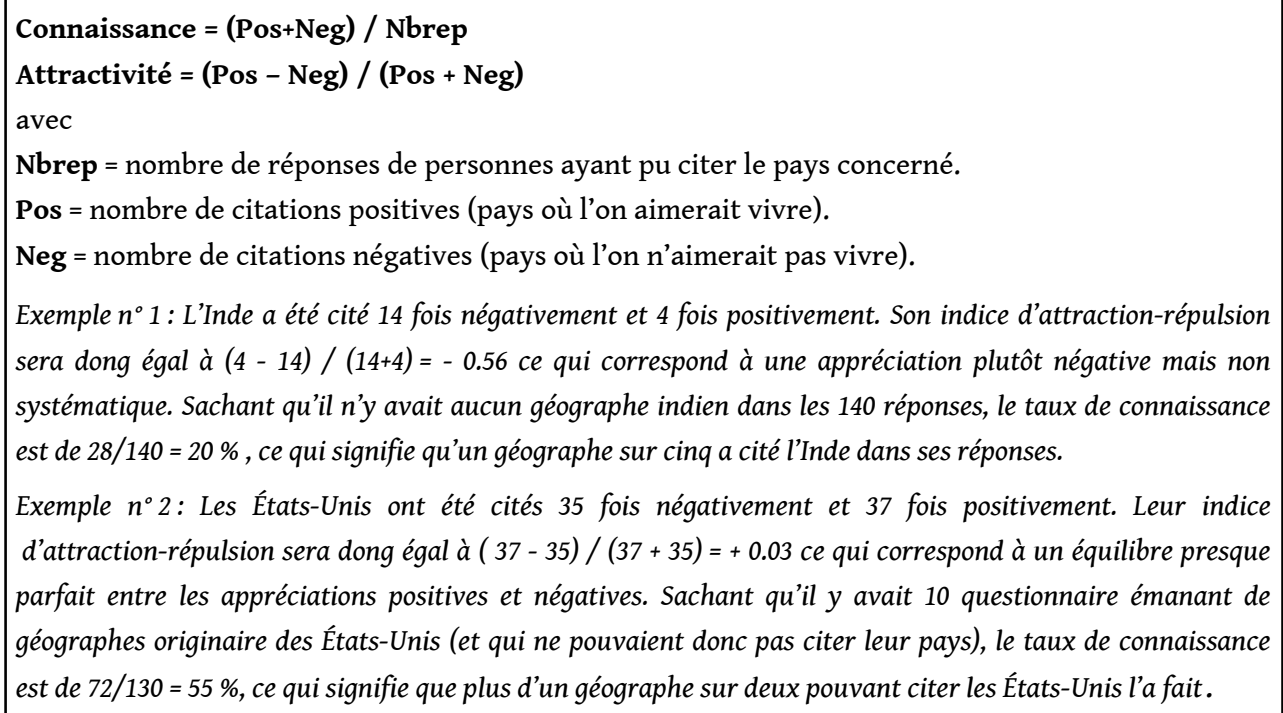

19 En combinant les deux indices, il est possible de visualiser simultanément sur un graphique les pays les plus connus (axe horizontal) et les pays les plus attractifs ou répulsifs (axe vertical). Le calcul de l'indice d'attractivité n'a cependant guère de sens lorsqu'il y a trop peu de réponses. C'est pourquoi le graphique n'est réalisé que pour les 50 pays ayant été cités par au moins 5 personnes (figure 2).

20 Cette figure offre un résumé synthétique de la vision du monde "moyenne" de l'échantillon de 140 géographes qui a été collectée lors du congrès UGI de Tunis. Mais il faut se garder d'en tirer des conclusions hâtives puisque, comme nous l'avons signalé, l'échantillon n'est aucunement représentatif de l'ensemble des géographes du monde et comporte une proportion très élevée de géographes tunisiens et européens. Or, il est visible que les citations de pays varient fortement selon l'origine géographique des personnes, comme on peut le voir sur la figure 3 qui montre les citations positives et négatives selon les cinq groupes de nationalité de résidence définis précédemment. La bonne approche consiste à étudier les variations du degré de connaissance et d'attractivité des pays en fonction de l'origine géographique des personnes enquêtées, en choisissant des couples de pays du monde ayant des caractéristiques voisines. Nous proposons d'illustrer cette méthode en comparant cinq binômes de pays: France/ Royaume-Uni, USA/Canada, Allemagne/Japon, Chine/Russie et Arabie Saoudite/Israël. 
Figure 2 - Connaissance et attractivité des pays du monde pour 140 membres du congrès UGI Tunis 2008

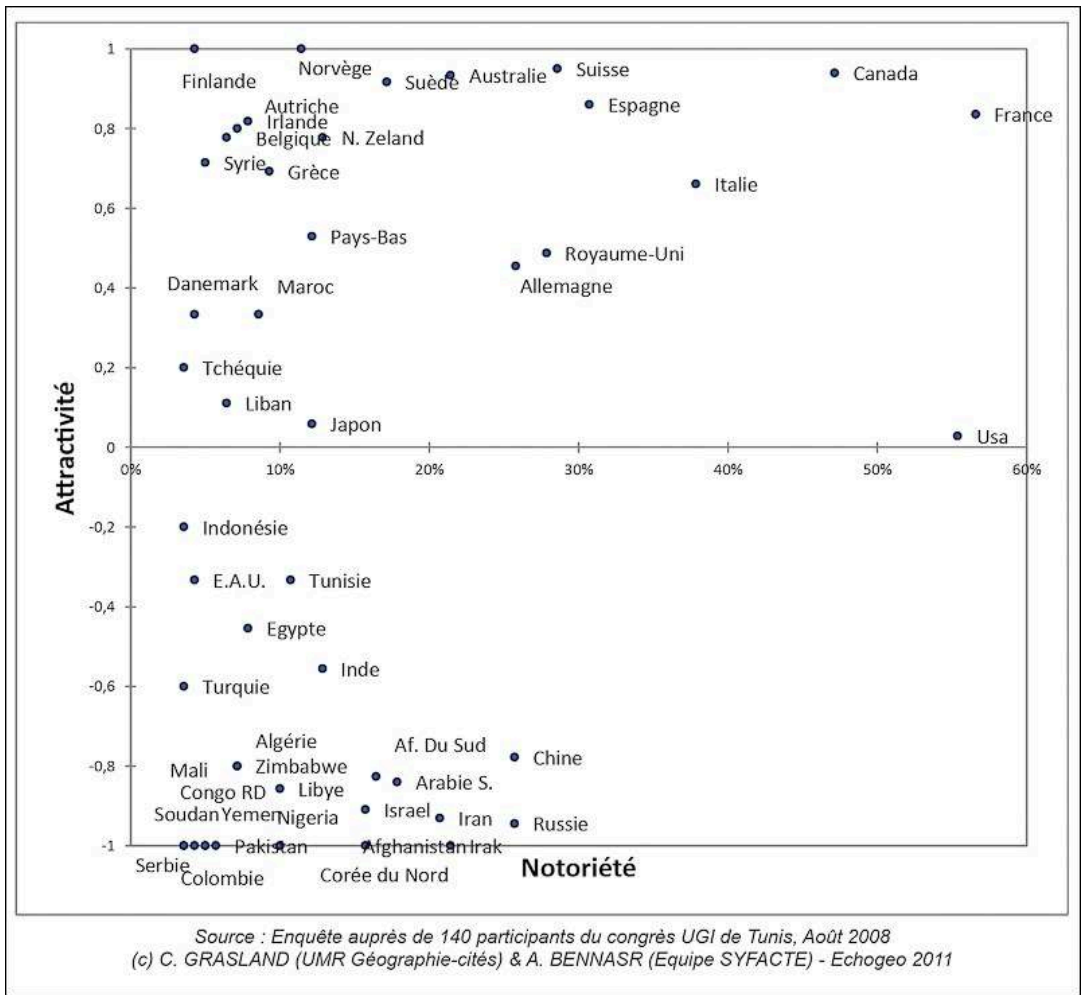

Source : Bennasr A. (Syfacte, Université de Sfax) \& Grasland C.(CNRS, Université Paris Diderot), enquête auprès de 140 participants du congrès UGI de Tunis, août 2008. 
Figure 3 - Distribution des pays cités par 140 membres du congrès UGI Tunis 2008 en fonction de leur origine géographique

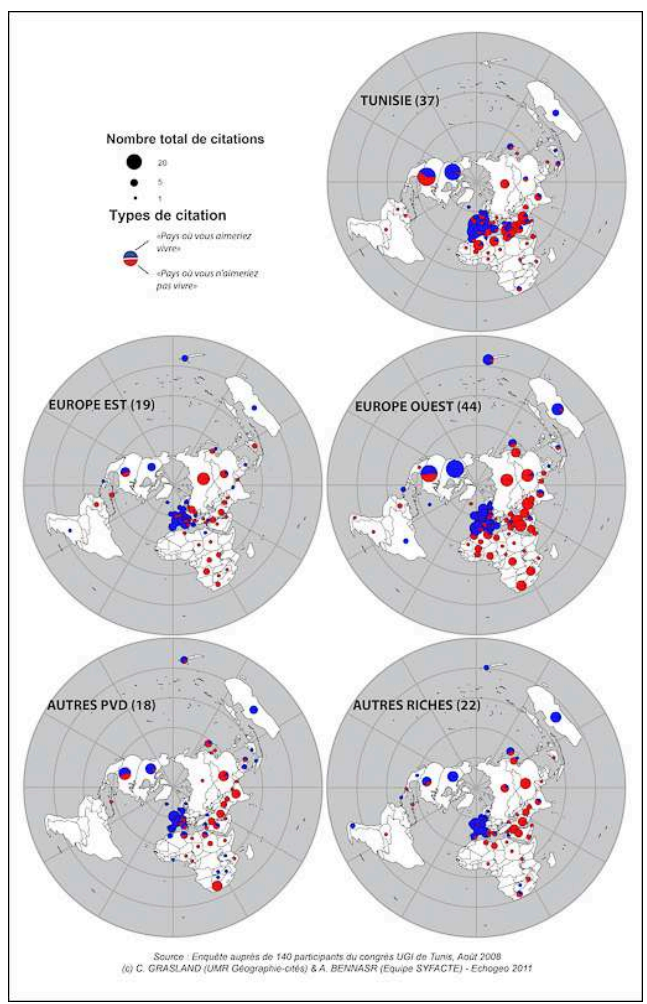

Source : Bennasr A. (Syfacte, Université de Sfax) \& Grasland C.(CNRS, Université Paris Diderot), enquête auprès de 140 participants du congrès UGI de Tunis, août 2008.

\section{Mise en évidence de la variabilité des perceptions selon l'origine géographique}

France et Royaume-Uni : le poids des langues et des héritages coloniaux

Tableau 4 - Perceptions comparées de la France et du Royaume-Uni

\begin{tabular}{|l|l|l|l|}
\hline FRANCE & Effectif & Connaissance & Attractivité \\
\hline Tunisie & 37 & $92 \%$ & 0,94 \\
\hline Europe de l'Est & 19 & $53 \%$ & 1,00 \\
\hline Europe de l'Ouest & 33 & $39 \%$ & 0,69 \\
\hline Autres Riches & 21 & $38 \%$ & 0,75 \\
\hline Autres PVD & 19 & $37 \%$ & 0,71 \\
\hline Ensemble & 129 & $56 \%$ & 0,86 \\
\hline
\end{tabular}




\begin{tabular}{|l|l|l|l|}
\hline ROYAUME-UNI & Effectif & Connaissance & Attractivité \\
\hline \hline Tunisie & 37 & $16 \%$ & 0,00 \\
\hline Europe de l'Est & 19 & $21 \%$ & 0,50 \\
\hline Europe de l'Ouest & 39 & $18 \%$ & 0,71 \\
\hline Autres Riches & 21 & $52 \%$ & 0,82 \\
\hline Autres PVD & 19 & $53 \%$ & 0,40 \\
\hline \hline Ensemble & 135 & $28 \%$ & 0,53 \\
\hline
\end{tabular}

Ce premier exemple permet de mettre en valeur l'importance des phénomènes historiques et linguistiques dans la perception des pays du monde. En considérant l'ensemble de l'échantillon, on pouvait avoir l'impression que la France était plus connue $(56 \%$ contre $28 \%)$ et plus attractive $(+0.86$ contre +0.53$)$ que le Royaume-Uni. Mais la décomposition des résultats par catégories d'origine montre une image beaucoup plus nuancée. En fait la France bénéficie du poids de l'échantillon tunisien qui la cite massivement ( $92 \%$ des réponses) et presque toujours positivement $(+0.94)$ alors que le Royaume-Uni est beaucoup moins connu (16\%) et apprécié $(+0.0)$ par les résidents de ce pays. Il en va un peu de même pour l'échantillon des géographes des pays d'Europe (Est ou Ouest) qui citent davantage la France que le Royaume-Uni et plus positivement, tout au moins en Europe de l'Est. Mais l'image change nettement lorsqu'on considère les pays plus éloignés qui citent le Royaume-Uni dans plus de la moitié de leur réponse alors que la France n'apparaît que dans 37 à $38 \%$ des cas. L'attractivité du Royaume-Uni est supérieure à celle de la France pour les géographes des pays riches et distants (USA, Australie, Canada, Israël) mais pas dans les pays émergents (Chine, Russie) où la France demeure plus attractive bien que moins citée.

\section{États-Unis et Canada : I'influence de la politique extérieure}

Tableau 5 - Perceptions comparées des États-Unis et du Canada

\begin{tabular}{|l|l|l|l|}
\hline USA & Effectif & Connaissance & Attractivité \\
\hline Tunisie & 37 & $68 \%$ & $-0,20$ \\
\hline Europe de l'Est & 19 & $32 \%$ & 0,00 \\
\hline Europe de l'Ouest & 44 & $48 \%$ & 0,05 \\
\hline Autres Riches & 11 & $64 \%$ & 0,43 \\
\hline Autres PVD & 19 & $63 \%$ & 0,17 \\
\hline Ensemble & 130 & $55 \%$ & 0,01 \\
\hline
\end{tabular}




\begin{tabular}{|l|l|l|l|}
\hline CANADA & Effectif & Connaissance & Attractivité \\
\hline Tunisie & 37 & $51 \%$ & 0,89 \\
\hline Europe de l'Est & 19 & $26 \%$ & 1,00 \\
\hline Europe de l'Ouest & 44 & $52 \%$ & 1,00 \\
\hline Autres Riches & 20 & $45 \%$ & 1,00 \\
\hline Autres PVD & 19 & $42 \%$ & 1,00 \\
\hline Ensemble & 139 & $46 \%$ & 0,97 \\
\hline
\end{tabular}

$\mathrm{Au}$ moment de l'enquête (Août 2008), l'image des États-Unis demeurait fortement marquée négativement dans beaucoup de pays du monde par la politique extérieure agressive de Georges W. Bush, tandis que le Canada bénéficiait d'une image positive d'ouverture aux migrations internationales et de relative tolérance. Il en résulte une différence frappante d'attractivité entre deux pays qui ont par ailleurs des taux de connaissance voisins ( $55 \%$ pour les États-Unis et $46 \%$ pour le Canada). Dans le cas du Canada, presque tous les avis sont positifs $(+0.97)$ puisqu'un seul géographe (tunisien) a déclaré ne pas vouloir vivre au Canada contre 63 autres qui ont déclaré vouloir y vivre... Le taux de citation est également très stable entre 40 et $50 \%$, même si les citations du Canada sont un peu plus faibles en Europe orientale. Le cas des États-Unis est très différent puisque l'indice d'asymétrie varie fortement d'un échantillon à l'autre. Les avis négatifs l'emportent clairement en Tunisie, tandis que la balance est équilibrée dans les pays européens et nettement positive dans le reste du monde, surtout dans les pays riches. On retrouve ainsi certaines caractéristiques du Royaume Uni, mais avec un niveau d'appréciation nettement moins favorable. Comme dans le cas du Canada, c'est en Europe de l'Est que le taux de citation des États-Unis apparaît le plus faible.

\section{Japon et Allemagne : attractivité économique et pesanteurs historiques}

Tableau 6 : Perceptions comparées de l'Allemagne et du Japon

\begin{tabular}{|l|l|l|l|}
\hline ALLEMAGNE & Effectif & Connaissance & Attractivité \\
\hline \hline Tunisie & 37 & $27 \%$ & 0,80 \\
\hline \hline Europe de l'Est & 19 & $37 \%$ & $-0,14$ \\
\hline Europe de l'Ouest & 32 & $28 \%$ & 0,33 \\
\hline Autres Riches & 21 & $10 \%$ & 1,00 \\
\hline \hline Autres PVD & 14 & $14 \%$ & 0,00 \\
\hline
\end{tabular}




\begin{tabular}{|l|l|l|l|}
\hline Ensemble & 128 & $23 \%$ & 0,40 \\
\hline
\end{tabular}

\begin{tabular}{|l|l|l|l|}
\hline JAPON & Effectif & Connaissance & Attractivité \\
\hline \hline Tunisie & 37 & $8 \%$ & 0,33 \\
\hline \hline Europe de l'Est & 19 & $0 \%$ & - \\
\hline Europe de l'Ouest & 44 & $11 \%$ & 0,20 \\
\hline Autres Riches & 20 & $25 \%$ & 0,20 \\
\hline \hline Autres PVD & 19 & $21 \%$ & $-0,50$ \\
\hline Ensemble & 139 & $12 \%$ & 0,06 \\
\hline
\end{tabular}

Ayant pour point commun d'être à la fois les grands perdants de la Seconde Guerre mondiale et des puissances économiques majeures du monde contemporain, l'Allemagne et le Japon offrent une image extérieure assez complexe. Si l'Allemagne paraît de prime abord plus connue que le Japon ( $23 \%$ de citations contre $12 \%$ ), c'est en grande partie du fait de la présence plus nombreuse des géographes européens et méditerranéens au congrès UGI de Tunis en 2008. En fait, chaque pays dispose d'une audience spécifique dans les pays qui lui sont le plus proches et c'est clairement le Japon qui est le plus cité dans les plus éloignés de l'Europe. Mais la proximité n'est pas un gage d'appréciation positive et on observe pour chaque pays que l'attractivité est souvent très faible dans les pays proches. Par exemple, les géographes d'Europe orientale sont ceux qui citent le plus l'Allemagne, mais un peu plus de la moitié déclarent qu'ils ne souhaiteraient pas y vivre. Il en va de même au Japon où les géographes chinois citent fréquemment le Japon, mais principalement comme lieu où ils ne souhaiteraient pas vivre. Bien que l'échantillon soit trop faible pour pousser davantage l'analyse, on peut émettre l'hypothèse que les héritages des conflits historiques demeurent encore très vivaces et nuisent à l'image extérieure de ces deux pays, pourtant caractérisés par un développement économique enviable.

\section{Chine et Russie : deux exemples de reconnaissance négative d'acteurs régionaux}

Tableau 7 : Perceptions comparées de la Chine et de la Russie

\begin{tabular}{|l|l|l|l|}
\hline CHINE & Effectif & Connaissance & Attractivité \\
\hline Tunisie & 37 & $5 \%$ & 0,00 \\
\hline Europe de l'Est & 19 & $21 \%$ & $-1,00$ \\
\hline Europe de l'Ouest & 44 & $27 \%$ & $-0,83$ \\
\hline
\end{tabular}




\begin{tabular}{|l|l|l|l|}
\hline Autres Riches & 21 & $38 \%$ & $-1,00$ \\
\hline Autres PVD & 15 & $53 \%$ & $-0,75$ \\
\hline Ensemble & 136 & $25 \%$ & $-0,82$ \\
\hline
\end{tabular}

\begin{tabular}{|l|l|l|l|}
\hline RUSSIE & Effectif & Connaissance & Attractivité \\
\hline \hline Tunisie & 37 & $14 \%$ & $-1,00$ \\
\hline \hline Europe de l'Est & 19 & $63 \%$ & $-1,00$ \\
\hline \hline Europe de l'Ouest & 44 & $27 \%$ & $-1,00$ \\
\hline Autres Riches & 21 & $24 \%$ & $-0,60$ \\
\hline \hline Autres PVD & 14 & $7 \%$ & $-1,00$ \\
\hline \hline Ensemble & 135 & $26 \%$ & $-0,94$ \\
\hline
\end{tabular}

24 Les deux grands pays communistes de l'époque de la guerre froide ont connu des évolutions considérables, tant sur le plan politique que sur le plan économique, depuis une vingtaine d'années. Bénéficiant d'une évidente notoriété, ils sont cités chacun par plus d'un quart des géographes de l'UGI. Mais cette notoriété est à l'évidence négative puisque c'est presque toujours comme pays où l'on ne souhaiterait pas vivre qu'ils sont mentionnés, le phénomène étant d'ailleurs encore plus marqué pour la Russie $(-0.94)$ que pour la Chine (-0.82). L'analyse détaillée des réponses par catégorie ne révèle pas de variabilité importante de l'indice d'attractivité, qui est presque toujours fortement négatif quelle que soit l'origine des géographes. Mais celui-ci montre en revanche que la connaissance de ces deux pays est extrêmement variable. Si la Russie est massivement citée dans les anciens pays socialistes d'Europe orientale (63\%) elle l'est beaucoup moins en Tunisie (14\%) et surtout dans les pays émergents éloignés de l'Europe $(7 \%)$. Le schéma est à peu près inversé pour la Chine qui est le plus fréquemment citée dans les pays éloignés de l'espace européen et méditerranéen, à la fois dans les pays riches ( $38 \%$ ) et plus encore dans les pays émergents (53\%). Dans l'un et l'autre cas, il faut insister sur le fait que le degré de connaissance élevé est à mettre en relation avec les aires d'influences économiques ou géopolitiques de ces deux grandes puissances. Le fait que l'appréciation soit négative (" pays où l'on n'aimerait pas vivre ») ne minore en rien le poids mental de ces pays dans les représentations du monde contemporain.

Israël et l'Arabie Saoudite : des conflits géopolitiques aux représentations 
Tableau 8 : Perceptions comparées d'Israël et de l'Arabie Saoudite

\begin{tabular}{|l|l|l|l|}
\hline ISRAEL & Effectif & Connaissance & Attractivité \\
\hline \hline Tunisie & 37 & $46 \%$ & $-1,00$ \\
\hline \hline Europe de l'Est & 19 & $0 \%$ & - \\
\hline \hline Europe de l'Ouest & 44 & $7 \%$ & $-1,00$ \\
\hline \hline Autres Riches & 16 & $6 \%$ & 1,00 \\
\hline Autres PVD & 19 & $5 \%$ & $-1,00$ \\
\hline \hline Ensemble & 135 & $16 \%$ & $-0,91$ \\
\hline
\end{tabular}

\begin{tabular}{|l|c|c|c|}
\hline ARABIE SAOUDITE & Effectif & Connaissance & Attractivité \\
\hline Tunisie & 37 & $16 \%$ & $-0,67$ \\
\hline Europe de l'Est & 19 & $5 \%$ & $-1,00$ \\
\hline Europe de l'Ouest & 44 & $23 \%$ & $-1,00$ \\
\hline Autres Riches & 21 & $24 \%$ & $-1,00$ \\
\hline Autres PVD & 19 & $16 \%$ & $-0,33$ \\
\hline Ensemble & 140 & $18 \%$ & $-0,84$ \\
\hline
\end{tabular}

Rapportés à leur poids économique, démographique ou géographique, peu de pays cristallisent autant l'attention des médias du monde entier qu'Israël et l'Arabie Saoudite. Situés au cœur d'enjeux politiques (conflit israélo-palestinien, terrorisme, ...) et économiques (accès aux ressources pétrolières) ces deux pays apparaissent de fait comme des éléments importants des représentations puisqu'ils sont chacun cités en moyenne par un géographe de l'UGI sur six. Comme la Russie et la Chine, ils sont surtout cités négativement à de très rares exceptions près, mais c'est la variation du taux de connaissance qui constitue l'élément intéressant. Israël a par exemple été mentionné 21 fois dont une seule positivement par un géographe américain. Or, parmi les citations négatives on trouve 17 géographes tunisiens et très peu de géographes européens ou du reste du monde, ce qui révèle une indifférence ou, plus probablement, une volonté de ne pas prendre position. La situation de l'Arabie saoudite est différente dans la mesure où le taux de citation est beaucoup plus régulier. Et cette fois-ci les géographes des pays occidentaux citent sans hésitation l'Arabie Saoudite comme pays où ils n'aimeraient pas vivre. 


\section{Essai de synthèse de la carte du monde des géographes de l'UGI}

Pour synthétiser les deux dimensions de notoriété et d'attractivité qui ont été dégagées dans les analyses précédentes, nous allons essayer de classifier les 50 pays qui ont été cités au moins 5 fois (positivement ou négativement) par les 140 géographes de notre échantillon. Chacun de ces 50 pays est alors décrit par 6 variables correspondant respectivement aux nombre de citations positives et au nombre de citations négatives de chacune des trois sous-populations de géographes de l'union européenne, de Tunisie et du reste du monde. Ce tableau de contingence va être analysé à l'aide d'une analyse factorielle des correspondances et d'une classification ascendante hiérarchique (Sanders, 1990). On sera ainsi en mesure de dégager les corrélations entre les visions du monde produites par les géographes de l'UGI en fonction de leur origine géographique (étude des axes factoriels) puis de construire une typologie des pays du monde en fonction de leur degré de connaissance et d'appréciation positive ou négative par chaque sous-groupe de géographes (classes résultant d'une classification fondée sur les corrélations entre profils de réponse, regroupés par la méthode du lien complet).

La première dimension structurante qui est révélée par l'analyse factorielle des correspondances (1er Axe, $49 \%$ de l'information) oppose sans réelle surprise les pays dans lesquels la plupart des géographes souhaiteraient vivre, quelle que soit leur origine géographique et les pays où personne ne souhaiterait vivre (figure 4).

Les appréciations positives les plus consensuelles concernent les pays scandinaves, les pays neufs, riches et ouverts à l'immigration (Australie, Canada, Nouvelle Zélande) ainsi que les pays d'Europe du Nord-Ouest (France, Belgique, Irlande) mais avec des nuances (le Royaume-Uni, l'Italie, les Pays-Bas, la Grèce ou l'Allemagne ont des scores un peu plus faibles que les précédents). On trouve également bien placés deux pays arabes : le Maroc et surtout la Syrie. L'effectif de citation de la Syrie est assez limité (7) mais on trouve deux appréciations positives par des géographes de l'union européenne, quatre par des géographes tunisiens et une seule citation négative par un géographe du reste du monde, en l'occurrence israélien. Pour le Maroc, on trouve 8 citations positives réparties équitablement entre géographes de l'union européenne (2), de Tunisie (4) et du reste du monde (2) et quatre citations négatives issues de l'union européenne (3) et de Tunisie.

Les appréciations négatives les plus consensuelles concernent quant à elles des pays ayant connu la guerre ou des troubles politiques récents, notamment des guerres internationales ou des guerres civiles (RD Congo, Pakistan, Soudan, Irak, Iran, Afghanistan, Yémen, Colombie), les pays connaissant ou ayant connu des régimes autoritaires (Corée du Nord, Zimbabwe), les grands pays émergents perçus comme des menaces politiques ou économiques (Russie, Chine, Inde, Arabie Saoudite) et enfin des pays d'Afrique subsaharienne comme le Nigéria, l'Afrique du Sud ou le Mali. D'autres pays comme l'Inde, l'Indonésie, la Tunisie, la Turquie, la Libye, l'Egypte, l'Arabie Saoudite et les Emirats Arabe Unis occupent une situation plus ambivalente puisqu'ils sont généralement cités négativement mais obtiennent tout de même une minorité de suffrages positifs.

Un nombre très limité de pays occupent une position centrale par rapport à cette dimension, ce qui signifie que les avis à leur sujet sont à peu près équitablement 
partagés dans toutes les régions du monde entre ceux qui souhaiteraient y vivre et ceux qui ne le souhaiteraient pas. On trouve évidemment dans cette situation les États-Unis mais aussi le Japon, la république Tchèque et le Liban.

Figure 4 - Les dimensions structurantes de l'appréciation des pays du monde par 140 géographes présents au congrès UGI de Tunis en août 2008

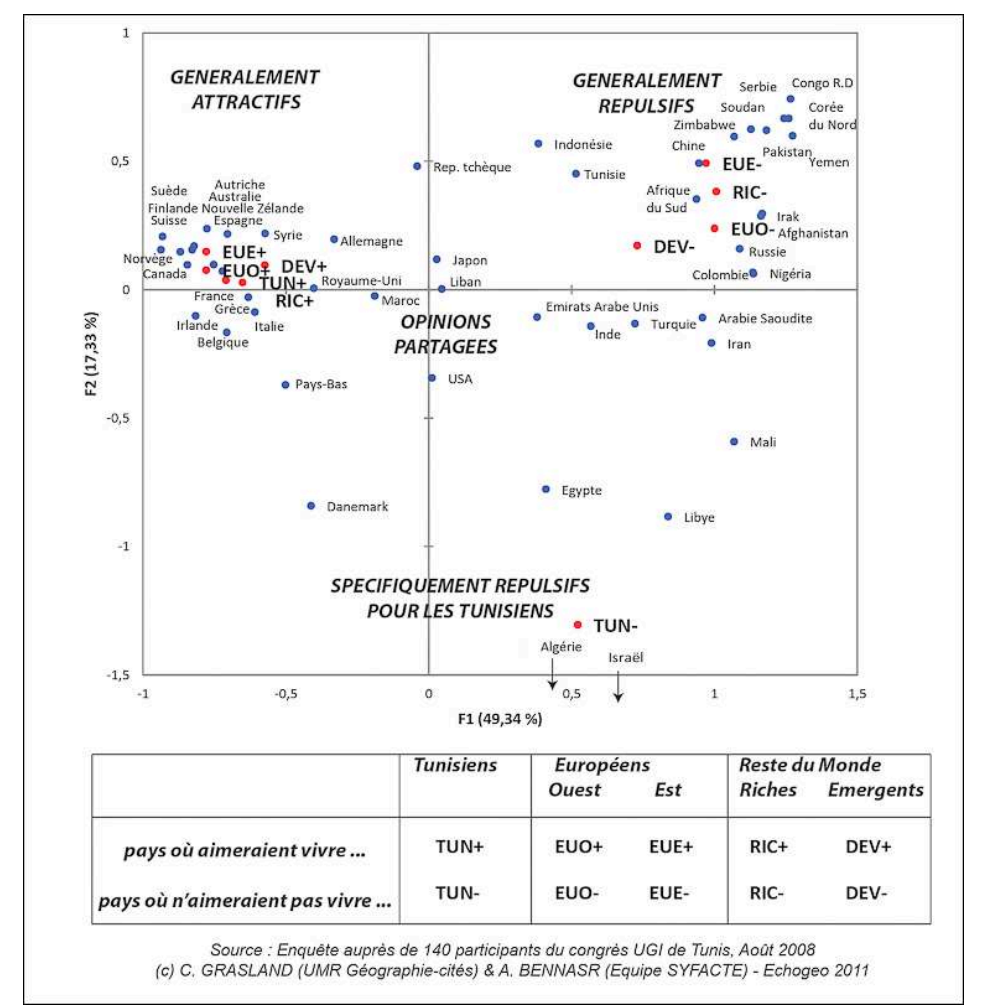

Source : Bennasr A. (Syfacte, Université de Sfax) \& Grasland C.(CNRS, Université Paris Diderot), enquête auprès de 140 participants du congrès UGI de Tunis, août 2008.

Au-delà de ce facteur global (au sens d'un consensus relatif au niveau mondial), on trouve sur les axes factoriels suivants des facteurs que l'on pourrait qualifier plutôt de « régionaux », puisqu'ils sont généralement surtout révélateurs des spécificités des réponses d'un des cinq groupes de géographes que nous avons définis. Le plus remarquable est le second axe factoriel (17\% de l'information) qui met en valeur les spécificités des réponses des géographes tunisiens en ce qui concerne les pays où ils ne souhaiteraient pas vivre. Israël et l'Algérie mais aussi, à un moindre degré, la Libye, L'Egypte, le Danemark, les Pays-Bas, les États-Unis, l'Arabie Saoudite et le Mali sont beaucoup plus fréquemment cités par les géographes tunisiens comme des pays où ils ne souhaiteraient pas vivre que par les autres géographes de l'union européenne et du reste du monde. Inversement, les géographes tunisiens ont moins tendance que les géographes de l'union européenne et du reste du monde à citer négativement des pays habituellement considérés comme les plus répulsifs par les autres régions du monde (Irak, Pakistan, Yémen, Inde, Chine, ...). Cela ne signifie pas qu'ils souhaitent vivre dans ces pays, mais ils ne constituent pas une priorité dans la liste des pays à citer comme ceux où l'on ne souhaiterait pas vivre. S'il existe à l'évidence une dimension régionale dans ces appréciations négatives (on cite davantage les pays voisins que l'on connait le mieux), il n'en demeure pas moins que d'autres composantes sont ici à l'œuvre, à la fois d'ordre politique (Algérie) et religieux (Israël, Iran, ...). L'affaire des caricatures du 
prophète, pourtant relativement ancienne au moment de l'enquête en août 2008, explique sans nul doute le fait que le Danemark ait été cité négativement par quelques géographes tunisiens. De la même manière, les politiques développées récemment aux Pays-Bas en matière de migration ont pu également nuire à l'attractivité de ce pays pour les Tunisiens.

Les axes factoriels suivants révèlent d'autres formes de spécialisation régionale moins importantes, tout au moins dans le cadre de notre échantillon de 140 géographes. Ainsi, l'axe 3 met en valeur des oppositions spécifiques entre les géographes des pays d'Europe centre orientale (qui citent massivement la Russie où l'Allemagne) et les géographes des pays émergents non européens (qui ne s'intéressent guère à la Russie mais beaucoup plus à l'Inde, le Japon ou l'Australie). Sans entrer dans le détail de ces axes, on insistera sur le fait qu'ils montrent plusieurs formes de différenciation « régionale » autres que celle qui opposait les géographes tunisiens au reste du monde sur l'axe 2. Si notre échantillon avait comporté moins de Tunisiens et plus de géographes d'Asie ou d'Amérique du Sud, il ne fait aucun doute que des spécificités propre à ces régions du monde auraient été révélées en lieu et place de celles des géographes tunisiens. Ces spécificités locales ne contredisent pas la coupure globale en pays attractifs et répulsifs de l'axe 1. Et la classification des pays en fonction des taux de citation positifs et négatifs des différents groupes de géographes permet de dégager clairement deux groupes de pays qui se subdivisent ensuite chacun en trois classes (figure 5).

Les pays où la plupart des géographes souhaiteraient vivre (type A) correspondent sans surprise aux pays les plus riches du monde (Europe de l'Ouest, Amérique du Nord, Australie, Nouvelle Zélande, Japon). Mais ils se différencient en trois sous-groupes distincts en fonction de la fréquence des citations qu'ils reçoivent des différents groupes d'origine géographique. Ainsi, le groupe A.1 (France, Espagne, Allemagne, Grèce, Maroc, Belgique, Liban, Syrie) apparaît beaucoup plus cité par les géographes tunisiens et, à un moindre degré, par les géographes d'Europe orientale. Le groupe A.3 (Royaume-Uni, Australie, Japon) suit un profil inverse et apparaît surtout massivement cité par les géographes des pays situés en dehors de l'aire européenne et méditerranéenne. Quant aux autres pays attractifs du groupe A.2 (USA, Canada, Italie, Suisse, Pays scandinaves, etc.), ils n'affichent pas de spécificités régionales particulières et apparaissent également attractifs pour tous les géographes quelles que soient leurs origines, avec toutefois une citation plus forte des pays d'Europe de l'Ouest.

Les pays où la plupart des géographes ne souhaiteraient pas vivre (type B) ont pour point commun de ne presque jamais être cités positivement, mais les avis négatifs qu'ils reçoivent obéissent à la même logique de spécialisation régionale que dans le type A. Le groupe B.3 (Israël, Libye, Egypte, Algérie, Mali) est ainsi clairement imputable aux déclarations des pays où les géographes tunisiens ne souhaiteraient pas vivre, tandis que le groupe B.2 (Russie, Congo RD, Colombie, Tchéquie, Indonésie, Turquie) reçoit surtout des avis négatifs en provenance des pays d'Europe centre orientale. Le groupe B.1 est plus universel (Chine, Irak, Iran, Arabie Saoudite, Corée du Nord, etc.) ou en tous les cas défini aussi bien par les géographes d'Europe de l'ouest que ceux de pays riches ou pauvres plus éloignés.

Les pays non cités par la plupart des géographes apparaissent en creux sur la carte du monde et constituent sans doute la découverte la plus cruelle pour la corporation des géographes. Ces derniers omettent en effet largement de citer la plupart des pays 
d'Amérique latine, d'Afrique subsaharienne, d'Europe centre orientale et d'Asie du sudest. Une partie de ces omissions est sans nul doute imputable à la composition de l'échantillon des géographes présents au congrès de l'UGI de Tunis en 2008. Mais cette explication est insuffisante car après tout certains pays ont été fortement cités alors même qu'aucun de leurs représentants n'était présent dans l'échantillon (Irak, Corée du Nord, Australie, ...). Le cas de l'Afrique est à cet égard symptomatique puisque l'on voit que les pays les plus riches ou les plus puissants y sont tout de même largement mentionnés (Egypte, Afrique du Sud, ...) tandis que les plus petits et les plus pauvres sont systématiquement oubliés. Sauf à être cités collectivement comme "pays africains » ou "Afrique subsaharienne » dans un certain nombre de réponses. Il faut sans doute y voir une marginalité croissante des recherches des géographes sur les «Suds des Suds », les financements étant de plus en plus concentrés sur les grands pays émergents par les opérateurs de financement des recherches au niveau international et national ...

Figure 5 - Typologie des pays du monde en fonction de leur notoriété et de leur attractivité pour 140 géographes présents au congrès UGI de Tunis en août 2008

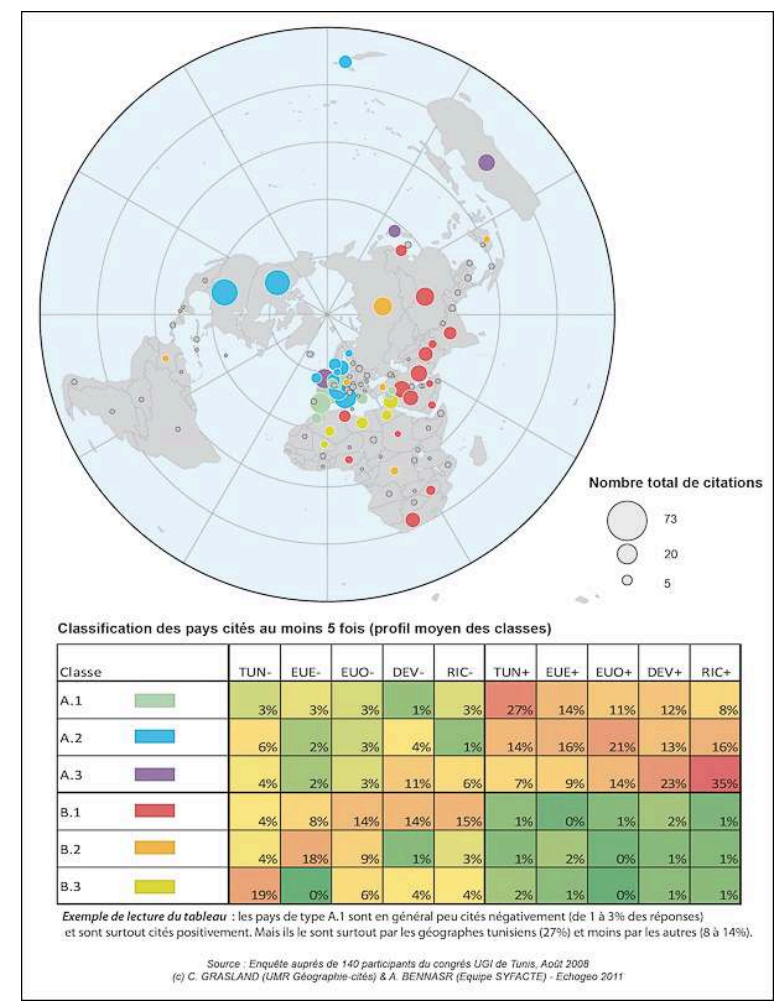

Source : Bennasr A. (Syfacte, Université de Sfax) \& Grasland C.(CNRS, Université Paris Diderot), enquête auprès de 140 participants du congrès UGI de Tunis, août 2008.

\section{Conclusion}

Même si les résultats obtenus au cours de cette enquête doivent être interprétés avec prudence, ils confirment que la vision du monde des géographes réunis au colloque UGI de Tunis en 2008 ne présente sans doute pas d'originalité extraordinaire par rapport à celle de la population des pays dans lesquels ils résident. Les géographes sont potentiellement plus à même que d'autres de citer des pays nombreux et éloignés, mais 
en pratique ils n'en concentrent pas moins leurs opinions sur les pays les plus grands et les plus proches de leur pays de résidence (Kolossov, 2003a). A l'instar des grands médias dont ils reflètent le discours tout en contribuant parfois à le nourrir (Kolossov, 2003b), les géographes apprécient les pays tolérants et ouverts sur l'étranger, si possible proches du leur en termes linguistique et culturel. Mais ils se méfient des voisins puissants ou qui ne partagent pas les mêmes valeurs.

En fait, les géographes présents au congrès UGI de 2008 ont tendance à obéir aux mêmes règles de connaissance et d'ignorance que les étudiants non géographes et à citer en priorité les pays les plus grands et les plus proches de leur lieu de résidence. Nous avons pu montrer dans le projet EuroBroadMap que le degré de connaissance des pays du monde par les étudiants obéit fondamentalement à une logique gravitaire et décroît avec la distance tout en augmentant avec la population et la surface (Grasland, Beauguitte, 2010). Dans le cas des citations positives, la richesse par habitant joue un rôle de correctif (on préfère évidemment vivre dans les pays à fort niveau de développement) et dans le cas des citations négatives c'est plutôt le poids médiatique (guerres, catastrophes, dictatures, ...) qui intervient comme facteur correctif. Certaines formes de proximité apportent des correctifs (frontières communes, langues communes, héritage colonial) mais ce sont tout de même les facteurs les plus banaux qui guident le choix des pays. Et les géographes professionnels de l'UGI n'échappent pas à la règle ...

En résumé, l'enfant amoureux de cartes et d'estampes n'a pas nécessairement un appétit égal à l'univers et n'est pas forcément plus à même que le commun des mortels de rêver de terres lointaines et inconnues.

\section{BIBLIOGRAPHIE}

ACA (Academic Cooperation Association), 2005. Perception of European higher education in third countries. Final Report, Project 2004-3924/001-001MUN-MUNA31.

Belhedi A., 1981. L'espace, subjectivité et perception : contribution à l'étude de la perception de l'espace. Revue Géographie et Développement, $\mathrm{n}^{\circ}$ 4, p. 7-37.

Blades M., 1990. The reliability of data collected from sketch maps. Journal of Environmental Psychology, $\mathrm{n}^{\circ}$ 10, p. 327-339.

Boyowa A. Chokor, 2003. Pattern of representations of countries I cognitive maps of the world with special reference to Africa. Journal of Environmental Psychology, 23,p. 427-437;

Cauvin C., 1999. Proposition pour une approche de la cognition spatiale intra-urbaine. Cybergeo, $n^{\circ} 72$.

Cauvin C., 2002. Cognitive and cartographic representations: towards a comprehensive approach. Cybergeo, $\mathrm{n}^{\circ} 206$.

De Singly F., 1992. L'enquête et ses méthodes : le questionnaire. Nathan Université, Paris. 
Didelon C., Grasland C., Richard Y., 2009. Atlas de l'Europe dans le monde. Paris, Documentation Française, $250 \mathrm{p}$.

Downs R.M., Stea B.D., 1973. Image and environment, cognitive mapping and Spatial behavior. Aldine Publishing Company. Chicago.

Downs R.M., Stea B.D., 1997.Des cartes plein la tête. Edison inc. St Hyacinthe, Québec.

Evan G.W., Marrero D.G., Butler P.A., 1981. Environmental learning and cognitive mapping. Environment and behaviour, 13, p. 1371-1388.

Fabry E., 2007. Les Européens face à la mondialisation. Kairos Future - Fondation pour l'Innovation Politique, Ministère des affaires étrangères, Paris, $332 \mathrm{p}$.

Golledge R.G., 1975.Cognitive configuration of the city, vol. 1. Ohio State Research foundation and Dept. of Geography, Columbus.

Gould P., White R., 1974. Mental maps. Penguin Books, New York/Baltimore.

Grasland C., Beauguitte L., 2010. Modelling attractiveness of global places: A worldwide survey on 9000 undergraduate students. Paper presented at the 50th European Congress of the Regional Science Association International on: Sustainable Regional Growth and Development in the Creative Knowledge Economy - 19th to 23th of August 2010 - Jönköping, Sweden.

Grataloup C, 2009. L'Invention des continents. Larousse, 224 p.

Kolossov V., 2003a. 'High' and 'Low' Geopolitics: Images of Foreign Countries in the Eyes of Russian Citizens. Geopolitics, 8:1, p. 121-148(28).

Kolossov V., 2003b. The world geopolitical vision in the mirror of newspapers and of public opinion. In Ilies A. and Wendt J. (Eds.), Europe between milleniums. Political Geography Studies, University of Oradea Press: Oradea, 2003, p. 11-22.

Kosslyn S.M. 1980. Image and Mind. Cambridge (Mass), Harvard University Press.

Liotta P.H., 2005. Imagining Europe: Symbolic Geography and the Future. Mediterranean Quarterly, p. $67-85$

Lynch K., 1960. The image of the city. Cambridge (Mass), MIT, Paris.

Moles A.A., Rohmer E., 1978. Psychologie de l'espace. Casterman, Paris Tournai.

Paulet J.P., 2002. Les représentations mentales en géographie. Anthropos-Economica, Paris, 152 p.

Robic M.C. ,Briend A.M., Rössler M., (sous la direction de), 1996. Géographes face au monde. L'Union géographique internationale et les Congrès internationaux de géographie. Paris, L'Harmattan, $464 \mathrm{p}$.

Robic M.C., 1997. Exclusion, régression...du “Ladies'Program ” au “ sans femme ” ?

Cybergeo, $\mathrm{n}^{\circ} 22$.

Saarinen T.F., 1987. Centering of mental maps of the World. Discussion paper, Department of geography and regional development, Tucson, Arizona.

Saarinen T.F., 1988a. Centring of mental maps of the world. National Geographic Research, 4 (1988), p. 112-127.

Saarinen T.F., Mac Cabe C.L., 1995. World Patterns of Geographic Literacy Based on Sketch map quality. Professional Geographer, 47(2), p 196-204.

Sanders L., 1990. L'analyse statistique des données en géographie. Montpellier, RECLUS (" Alidade »), $267 \mathrm{p}$. 
Spencer R., Blades M., 1986. Pattern and process: a review essay on the relationship between behavioral geography and environmental psychology. Progress in Human Geography, vol. 10, $\mathrm{n}^{\circ} 2$, p. 230-248.

Walker M., 2000. Variable Geography: Mental maps of a Greater Europe. International Affairs, 76, 3,459-474.

Zheng L-H, Desjeux D., Boisard A-S., 2003. Comment les Chinois voient les Européens, PUF, Collection Sciences sociales et sociétés, $145 \mathrm{p}$.

\section{NOTES}

1. http://www.eurobroadmap.eu

\section{RÉSUMÉS}

140 des 1000 géographes présents au congrès UGI de Tunis d'Août 2008 ont accepté de répondre à une enquête sur leur carte mentale du monde. Le présent article propose d'étudier leur vision du monde à travers les réponses à une question leur demandant de citer, en dehors de leur propre pays, un à cinq pays où ils aimeraient vivre et un à cinq pays où ils n'aimeraient pas vivre. L'analyse des résultats est fondée sur la mesure de la connaissance (fréquence de citations) et de l'attractivité (balance des opinions positives et négatives) des pays du monde et sur la comparaison des résultats obtenus par sous-ensemble de géographes. Le principal résultat, inattendu, est la relative banalité des réponses obtenues: les géographes professionnels ne semblent pas citer des pays particulièrement originaux et privilégient les plus grands et les plus proches de leur lieu de résidence. Il existe toutefois des variations intéressantes dans les lieux cités lorsque l'on compare les réponses des géographes tunisiens, européens et du reste du monde, surtout pour les pays où ils ne souhaiteraient pas vivre.

140 out of 1000 geographers present at the IGU Congress of Tunis (August 2008) has agreed to answer to a survey on their mental map of the World. The paper propose to analyze their vision of the world on the basis of their answers to the question "Except your own country, quote 1 to 5 countries you would like to live in and 1 to 5 countries where would not like to live in". The analysis of result is based on the joint analysis of knowledge (countries that are the most frequently mentioned) and attractiveness (balance of positive and negative opinion) by subsamples of geographers. The main result -unexpected-is the fact that the answers are really not original: professional geographers are mainly quoting the most important countries of the world and the countries located at short distance from their place of residence. It is nevertheless possible to point interesting differences between the answers of geographers from Tunisia, Europe and the rest of the world, especially concerning countries where they would not like to live. 
INDEX

Mots-clés : attractivité, carte mentale, géographie, monde, pays, représentation, Tunisie, UGI

Keywords : attractiveness, geography, IGU, mental map, representation, states, Tunisia, world

\section{AUTEURS}

\section{ALI BENNASR}

Ali Bennasr est Maître de conférences à l'Université de Sfax et membre du laboratoire Syfacte, Sfax, Tunisie. abennasr@hotmail.fr

\section{CLAUDE GRASLAND}

Claude Grasland est Professeur des universités, Université Paris Diderot et membre de CNRS-UMR 8504 Géographie-cités. claude.grasland@parisgeo.cnrs.fr 